$16^{\text {th }}$ International Congress of Metrology, 02009 (2013)

DOI: $10.1051 /$ metrology/201302009

(c) Owned by the authors, published by EDP Sciences, 2013

\title{
Standard for High Viscosity and Multiphase Liquid Flow Measurement
}

\author{
Wu Jian ${ }^{1}$ and Luo Rongmo ${ }^{1, \text { a }}$ \\ ${ }^{1}$ National Metrology Centre (NMC), A*STAR. 1 Science Park Drive, Singapore 118221.
}

\begin{abstract}
Mass flow meters are deployed for metering of heavy hydrocarbon such as bunker fuels. There are many challenges in the measurement of mass flow influenced by high viscosity and multiphase fluids. A primary liquid flow standard has been developed and is described in this paper. This standard is capable to perform water and high viscous fluid calibration from 0.6 to $80 \mathrm{Mt} / \mathrm{h}$ using gravimetric method, with two phase flow condition in the range of 0 to $30 \%$ gas-liquid volume ratio and measurement uncertainties range of $0.05 \%$ to $0.2 \%$.
\end{abstract}

\section{Introduction}

Fuel oil is described as heavy oil because of high density and high viscosity. In fuel oil production and transferring pipelines, challenges of metering heavy oil are normally encountered for its ready entrainment of gas and difficulties in separating gas from the oil [1-3]. Accurate two-phase measurement of heavy oil is therefore a desirable technical goal. To meet the requirements on more accurate measurements of the viscous heavy oil streams for allocation and custody transfer purposes, some innovative measurement methods, such as ultrasonic flow meter, electromagnetic flow meter, are investigated. The Coriolis mass flow meters are relatively modern flow measurement technologies and continue to capture increasing market share as operators gain confidence in their performance [4].

This paper describes a liquid flow measurement primary standard. This standard is disseminated using gravimetric calibration directly traceable to the unit of mass and the reference standard of Coriolis flow meters. The set-up of this facility has four parallel pipelines with diameters of $0.5,2,3$ and 4 inches with multiple connection structure which allows bi-directional flow. Two weighing systems with capacities of $32 \mathrm{~kg}$ and 600 $\mathrm{kg}$ are able to calibrate water flow meters from 0.6 to 80 $\mathrm{Mt} / \mathrm{h}$. Another pair of similar weighing system is also set up for the viscous fluid calibration in the same flow ranges, which is capable of comparing different viscous fluid measurements directly against water flow calibration, hence the influence due to different fluid properties can be identified, quantified and compensated.

\section{Static gravimetric method}

Static gravimetric liquid flow calibration systems are widely used as primary liquid flow standards. Primary static weight flow calibrations are performed by collecting a measured mass of the fluid flowing through the meter being calibrated under stable conditions of flow, pressure, and temperature. The quantities measured in connection with the calibration such as temperature, mass, time, and density are traceable to NMC's reference or primary standards. Cumulated mass flow through the

\footnotetext{
a Corresponding author: luo_rongmo@nmc.a-star.edu.sg
}

meter is calibrated against the mass collected in the weighing tanks, with correction for buoyancy effects and the residue value in pipelines as,

$$
m=M * F+K * V * d p
$$

where $\mathrm{M}$ is the collected mass. The inventory volume, $\mathrm{V}$, is the volume of piping between the meter under test and the standard to measure the flow, which is located at the end of the pipe. $F$ and $K$ are the correction factors for buoyancy and residue volume in the pipeline. The $d p$ is the pressure difference between steady flow process and end of the collection interval.

\section{The primary flow standard}

The set-up of the flow standard is shown in Figure 1. The system is a closed loop flow system consisting of two pumping systems. A screw pump with maximum 100 $\mathrm{m}^{3} / \mathrm{h}$ capability is used to generate flow for water flow calibration. A bypass way with adjustable valves is arranged at downstream of the pump to vary the flow rate at wide ranges. A higher capacity gear pump is to generate the flow with high viscous fluid up to $1000 \mathrm{cSt}$. Both water and high viscous liquids are able to flow through the meter under test with bi-directions without changing its setting up. This arrangement allows eliminating the influences caused by the different strains forces in each calibration set-up and enhances the system to precisely analyse the difference due to viscous fluid properties. Multiple valves are arranged to configure flow direction and flow rates to cater the full range of flow measurements and satisfy the requested flow conditions. At least five PTR thermal meters and pressure measurement at different locations across the meter and pipelines to monitor the flow conditions and stability as well as compensate for head pressure lost. A controllable heater is installed to adjust the temperature of viscous fluid, so that the meter can be characterized according to the field conditions. In addition, the desired fluid viscosity can be obtained for calibration using an in-line viscometer. 
Figure 1. NMC's primary multiphase - high viscosity liquid flow standard

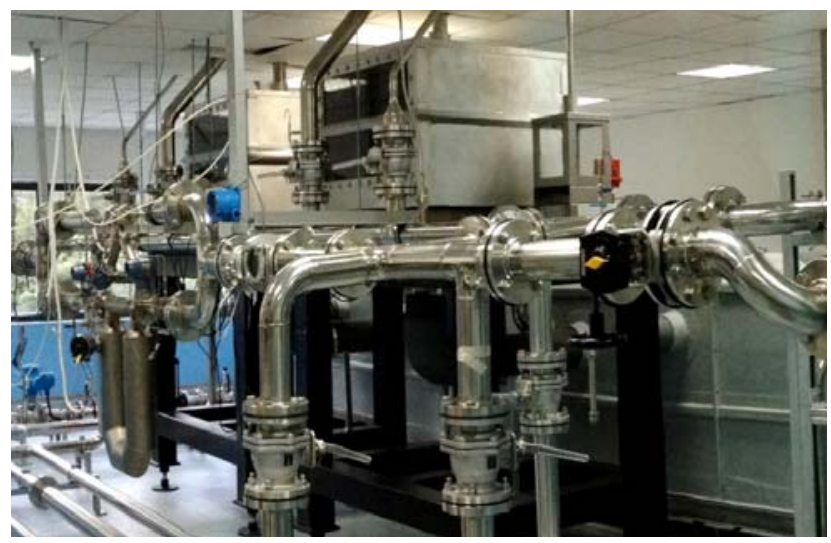

\section{System integration}

The entire calibration facility is integrated by in-house software based on the LabView language. The interfacing cards are able to support 20 PRT probes and also able to collect signal from pressure sensors, temperature sensors, Coriolis flow meters and weighing scales. The control signal to the valves allows automatically operating the valves according to preset calibration procedures. Furthermore, instant viscosity measurement allows morning the fluid property during the calibration intervals. Thereafter the integration for heater, pump speed and flow rate control valves can be integrated also for fine tuning flow setting as well as for better flow conditions.

\section{Function for multiphase flow measurement}

The system was designed to perform the calibration of mass flow with gas injection. A gas flow injection system with high pressure and measured gas flow rate were built in connection with the pipeline at upstream of the meter under test. Different gas void fraction (GVF) can be generated by measuring liquid flow rate, gas flow rate and the pressure in the pipeline. The two phase flow is able to be observed at windows located before and after the meter as shown in Figure 2. Since the gravimetric flow method has being used, the gas void mass flow rate can be accurately measured without being affected by the presence of gas, and hence the deviation of meter due to this reason can be known and corrected. Currently, the system is able to perform void ratio up to $30 \%$. It is possible to increase to $50 \%$ and even more at lower liquid flow rate. Most mass flow meters, however, are not able to indicate the actual rate at such high gas-liquid void ratio.
Figure 2. Gas-Liquid two phase flow measurement

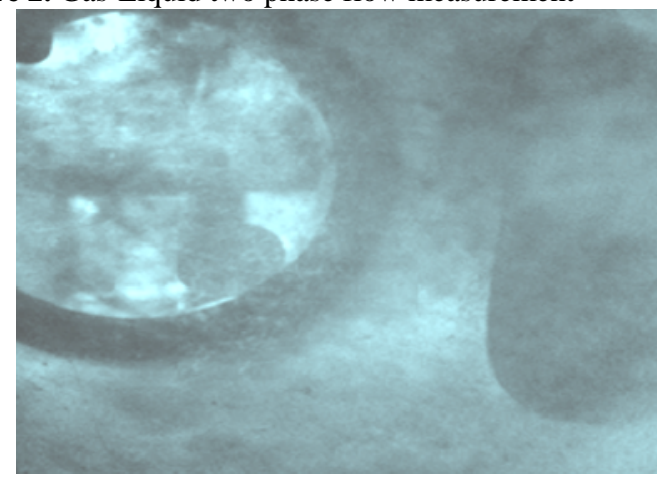

\section{High viscosity fluid measurements}

One of the main functions of the calibration facility is to perform high viscosity fluid flow calibration. The unique design of the standard set-up allows calibration of mass flow meter with the medium viscosity up to $1000 \mathrm{cSt}$. Due to the same reason for adopting the gravimetric method, this facility is able to perform measurements without significantly increasing measurement uncertainty and there is no deviation due the viscosity effects in principle. The meter can be characterized with comparison against with the water calibration results by the same flow configuration and setup via the gravimetric method also.

\section{The measurement corrections}

\subsection{Buoyancy}

Liquid mass values observed by the weighing scales was calibrated which has different buoyancy force to the stainless mass. To obtain the true mass of the collected liquid, measurement of air and liquid fluid densities are required. The mass at standard condition can be found using the following relationship:

$$
M_{S}=\frac{\rho_{W}-\rho_{S}}{\rho_{W}-\rho_{a}} \cdot M
$$

where $M$ is the apparent mass indicated by the scale, $\rho_{a}$ the air density, $\rho_{S}$ is the density at standard condition and $\rho_{W}$ is the liquid density. The buoyancy uncertainty for the liquid flow calibration was estimated less than $0.001 \%$.

\subsection{Leakage and splashes}

Liquid splashing out from weigh collection system and minor leaks from the pipework are usually apparent and are trimmed off before calibrations start. Therefore, uncertainties for splashes or leaks are neglected.

\subsection{Residue volume}

Storage effects arise due to changes in the pressure during the measurement process at the start and end stages. The pressure changes in the system indicate that 
changes in density of the water and dimensions of the pipes for the volume between meter under test and the outlet of the fishtail varying accordingly. The quantity due to storage effects must be considered and properly corrected. The correction factor is based on experiment with different pipeline length and characterized as:

$$
V=K *\left(P_{1}-P_{0}\right) * \sum_{i}\left(\pi L_{i} r_{i}^{2}\right)
$$

where $P_{1}$ is the pressure at flow condition and $P_{0}$ is atmosphere pressure, $L$ is the total length of the pipeline between meter under test and the outlet of the fishtail, and $r$ is the radius of the pipe. With proper compensations, this effect is estimated to be less than $0.007 \%$

\subsection{Water evaporation}

In the case of longer collection time, evaporation of water became a factor to cause measurement deviations. To minimise the error to an acceptable level, a correction factor has been applied to the gravimetric process on the basis of ambient pressure, temperature, as well as the humidity. Typically the single measurement can be done within $15 \mathrm{~min}$ to fill the tank completely. A set of experiments show that the evaporation is less than $0.001 \%$ under the laboratory calibration conditions.

\subsection{Liquid density}

In order to perform necessary conversion from mass to volumetric flow the fluid density must be known. Density of the liquid flowing through the meter being calibrated is normally required. The liquid density is able to be measured in the laboratory and a compensation factor can be applied according to fluid temperature at the metering position. The measurement uncertainty for water density is about $0.003 \%$. With unstable temperature limited within $0.2^{\circ} \mathrm{C}$ during a measurement, the expanded uncertainty is about $0.008 \%$.

\section{Summary of Measurement Uncertainty}

\subsection{The mass balance}

The calibration of mass balance can be carried out on site after removed the water tanks, and the measurement uncertainty due to mass is less than $0.0005 \%$. The $600 \mathrm{~kg}$ weighing scale was modified to allow drain pipes and control valves allocated at the center positions, so that the off center effects is insignificant. With the repeatability, resolution and linearity of scale, the overall estimation of the measurement uncertainty is about $0.006 \%$. In considering off center arrangement for $32 \mathrm{~kg}$ weighing tanks, the measurement uncertainty is about $0.008 \%$ of a reading.

\subsection{The repeatability of gravimetric method}

The repeatability of liquid collection is affected by the liquid inlet section, process flow and pressure stability, leakage and splashes. Tests show that the entire system is able to perform a reasonable repeatability within $0.025 \%$. And the summary measurement uncertainty is listed as Table 1.

Table 1. The summary measurement uncertainty

\begin{tabular}{|c|c|c|c|}
\hline Items & Symbol & Value & $\begin{array}{l}\text { Standard } \\
\text { Uncertainty }\end{array}$ \\
\hline - Resolution & $\mathrm{R}_{\mathrm{s}}$ & $\begin{array}{l}0.1 \mathrm{~g}- \\
10 \mathrm{~g}\end{array}$ & $0.001 \%$ \\
\hline $\begin{array}{l}\text { - Weighing scale } \\
\text { calibration }\end{array}$ & $\mathrm{U}_{\mathrm{cal}}$ & $\begin{array}{c}0.006 \\
\%\end{array}$ & $0.003 \%$ \\
\hline $\begin{array}{l}\text { - Reproduceabili } \\
\text { ty of weighing } \\
\text { scale } \\
\end{array}$ & $\mathrm{R}_{\mathrm{e}}$ & $\begin{array}{c}0.005 \\
\%\end{array}$ & $0.0028 \%$ \\
\hline - Buoyancy & $\overline{\mathrm{U}_{\mathrm{b}}}$ & $\begin{array}{c}0.001 \\
\%\end{array}$ & $0.0006 \%$ \\
\hline $\begin{array}{l}\text { - Leakage and } \\
\text { Splashes }\end{array}$ & $\overline{\mathrm{U}_{\mathrm{l}}}$ & - & - \\
\hline $\begin{array}{l}\text { - Residue } \\
\text { Volume: }\end{array}$ & $\mathrm{U}_{\mathrm{v}}$ & $\begin{array}{c}0.007 \\
\%\end{array}$ & $0.004 \%$ \\
\hline $\begin{array}{l}\text { - Water } \\
\text { Evaporation }\end{array}$ & $\mathrm{U}_{\mathrm{e}}$ & $\begin{array}{c}0.001 \\
\%\end{array}$ & $0.001 \%$ \\
\hline - Liquid Density & $\rho$ & $\begin{array}{c}0.008 \\
\%\end{array}$ & $0.008 \%$ \\
\hline - Repeatability & $\mathrm{R}_{\mathrm{p}}$ & $\begin{array}{c}0.025 \\
\%\end{array}$ & $0.025 \%$ \\
\hline $\begin{array}{l}\text { Combined } \\
\text { uncertainty }\end{array}$ & & & $0.026 \%$ \\
\hline $\begin{array}{l}\text { Expanded } \\
\text { uncertainty }\end{array}$ & & & $0.05 \%$ \\
\hline
\end{tabular}

\section{Conclusion}

The established flow standard was successfully developed for calibrations of mass flow meter at low and medium liquid flow range. The facility is not only able to perform conventional water flow calibration, but also able to perform the calibration according to varying fluid viscosity and field conditions. It gives an added advantage in characterizing the viscous effects of mass flow meter and two phase flow.

\section{References}

1. S. Al-lababidi, A. Addali, H. Yeung, D. Mba, F. Khan, J. Vib. Acoust. , 131(6), 064501 (2009).

2. M. Henry, M. Tombs, M. Duta, F. Zhou, R. Mercado, F. Kenyery, J. Shen, M. Morles, C. Garcia, R. Langansan, Flow Meas. Instrum., 17(6), 399-413 (2006).

3. F. Dong, F.S. Zhang, W. Li, C. Tan, J. Phys. Conf. Ser., 147(1), 012044 (2009).

4. M. Henry, M. Tombs, M. Zamora, F. Zhou, Flow Meas. Instrum., 30(0), 112-122 (2013). 